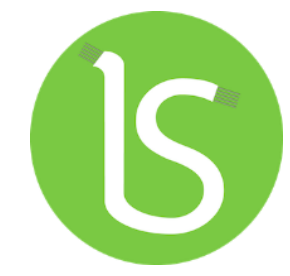

\title{
Estrategias de marketing en tiempos de Covid-19 en Empresas del sureste mexicano
}

\author{
Carlos David Zetina Pérez a \\ Sandra Juárez Solís b \\ Leidy Cristhel Alvarado García c
}

Resumen - Los cambios constantes en el entorno son un fuerte reto que hace necesaria la toma de decisiones y en este año de 2020 sin duda alguna la situación de la pandemia COVID-19 afectó a todos los sectores en un evento histórico que pone en riesgo el funcionamiento de un gran número de empresas en el mundo, de manera importante el marketing estratégico brinda la dirección para enfrentar los cambios y ayuda a conocer las estrategias enfocadas desde el Marketing Mix. El estudio llevó a cabo 26 entrevistas con empresarios de zonas urbanas y rurales in Situ, además se aplicó un cuestionario tipo Likert a 38 empresas, todo esto con la finalidad de hacer una descripción de la afectación y de las principales estrategias concluyendo que determinados tipos de negocio y en zonas rurales el impacto fue menor, siendo la plaza la estrategia del Marketing Mix más socorrida.

Palabras clave - Marketing Mix, Empresas, Covid, Estrategias, Tendencias.

Abstract - The changes in the environment are a strong challenge that makes take a decision necessary in this year of 2020. The situation about Pandemic COVID-19 affected all sectors in a historic event that puts the operation of many companies in the world at risk, in an important way, strategic marketing provides the direction to face the changes and helps to know the strategies focused from the Marketing Mix. The study carried out 26 interviews with entrepreneurs from urban and rural areas in Situ, in addition a Likert-type questionnaire was applied to 38 companies, all with the purpose of making a description of the affectation and the main strategies, concluding that certain types of business and in rural areas the impact was less, with the market being the most helpful Marketing Mix strategy.

Keywords - Marketing Mix, Companies, Covid, Strategies, Trends.

\section{CÓMO CITAR \\ HOW TO CITE:}

Zetina-Pérez, C. D., JuárezSolís, S., y Alvarado-García, L. C. (202I). Estrategias de marketing en tiempos de Covid-19 en Empresas del sureste mexicano. Interconectando Saberes, (12), 89-99.

https://doi.org//0.25009/is.v0il2 .2702

Recibido: 13 de abril de 202 I Aceptado: 5 de julio de 202I Publicado: 20 de julio de 2021

a Universidad Juárez Autónoma de Tabasco, México. E-mail: carzeti@hotmail.com

(iD) 0000-0003-0397-9157

b Universidad Juárez Autónoma de Tabasco, México. E-mail: marketingvhsa@outlook.com

(iD) 0000-0002-7883-3622

c Universidad Juárez Autónoma de Tabasco, México. E-mail: marketingvhsa@outlook.com 


\section{INTRODUCCIÓN}

Los cambios constantes en el entorno representan para las empresas un reto fuerte y continuo que hace necesaria la toma de decisiones para adaptarse al mundo complejo (Burbano-Pérez, 2017).

Dichos cambios o alteraciones en el ambiente exigen que las empresas deban adaptar sus estrategias y alterar sus estructuras organizacionales (Pérez-Vallejo, Vilariño-Corella y Ronda-Pupo 2017).

El año 2020 trae un evento histórico trascendental afectando a todos los sectores, industrias y actividades sociales y la Organización Mundial de la Salud (OMS) ha clasificado la situación COVID-19 como una pandemia (Gomar, 2020).

La pandemia por SARS-CoV-2 llega a todo el mundo con amplia mortalidad e impacto social afectando a un gran número de personas propiciando que las actividades se desarrollen en confinamiento (Matias-Guiu, Gómez-Pinedo, Montero-Escribano, Gómez-Iglesias, Porta-Etassam y Matias-Guiu 2020).

El 24 de marzo de 2020 se publica en el Diario Oficial de la Federación el acuerdo para tomar las medidas preventivas para la mitigación y control de los riesgos para la salud definidas en la "Jornada Nacional de Sana Distancia que tiene como objetivo el distanciamiento social, así mismo indica que se debe evitar la asistencia a centros de trabajo personas en condiciones vulnerables y suspender temporalmente las actividades que involucren concentración física, tránsito o desplazamiento de personas entrando en vigor del 2020 así como instrumentarse planes (Instituto Nacional Electoral, 2020).

Esta emergencia menciona Guevara (2020) pone además en riesgo el funcionamiento de un gran número de empresas en el mundo, haciendo notoria la importancia de adaptarse ante el cambio de acuerdo con García-Muñoz, Sánchez y Navarrete (2020) e implementar nuevas estrategias adaptándose además a la incertidumbre que desarrolla habilidades para manejar nuevas tecnologías ya que muchas organizaciones no han parado su producción y sus trabajadores realizan su labor desde casa.

\section{DESARROLLO}

La administración en las organizaciones debe interpretar los objetivos propuestos por la organización y transformarlo en acción organizacional a través de la planeación, la organización, la dirección y el control de las actividades realizadas en las áreas de la empresa (Marín Portillo, K., y Atencio Cárdenas, E., 2008).

Ante el riesgo y las medidas preventivas dictadas por las autoridades sanitarias las empresas se adaptan a las circunstancias del COVID-19 con diferentes medidas como adoptar nuevas formas de trabajo y cristalizando diferentes ideas de emprendimiento para generar recursos, utilizando diversas plataformas, de esta manera los sectores tradicionales han tenido que adaptarse a los cambios (Zabaleta y Gaona, 2020).

En esta situación dialéctica del entorno con la empresa DAnsoff y Leontiades (1976) mencionan se debe hacer una reorientación periódica que para deshacer la miopía de mercado implementando la técnica llamada planificación estratégica que implica la combinación del entorno con la empresa y esto ayuda menciona Montes (201l citado por Peñafiel-Loor, Pibaque-Pionce y Pin-Sancan 2019) a la toma de decisiones de una manera rápida y establece objetivos a futuro para mejora de la organización adaptándose a los cambios en el entorno. 
Los modelos de planeación estratégica más comunes determinan la visión, misión y los objetivos de mediano y largo plazo, así como la formulación de las estrategias y cursos de acción necesarios para alcanzar las metas (Ruiz, Aguilar, García, González, Vega y Velandia 20II).

Dichas estrategias son el enlace entre las habilidades y recursos internos de la empresa con las oportunidades y amenazas creadas por sus factores externos (Ascencio, Campo, Ramírez y Zapata 2016).

Entre las estrategias de las empresas para gestionar la adaptación a un entorno cambiante fundamentado en la fijación de objetivos, el análisis de las oportunidades, la fijación de estrategias, la implementación y control se encuentra el marketing estratégico (Sukier, Hernández-Fernández, PortilloMedina, Valle-Ospino, García y García-Guiliany 2018).

El marketing es una actividad que lleva realizándose desde hace mucho tiempo atrás, tradicionalmente estaban orientadas a las ventas en mercados masivos pero la situación y evolución del mercado obliga a segmentar y a realizar continuamente estudios del consumidos y centrar los esfuerzos en satisfacer sus necesidades de la mejor forma posible (Cruz, 20I5).

La importancia del marketing estratégico es proporcionar una visión clara del objetivo final y su definición contiene las estrategias de cartera, estrategias de posicionamiento, estrategia de segmentación, estrategia funcional (Marketing Mix), estrategia de crecimiento y estrategia de fidelización de clientes (Espinoza, 2016 citado por Saldarriaga y Contreras, 2018).
En 1960 Jerome McCarthy introduce "La clasificación de las 4P's del Marketing Mix, producto, precio, plaza y promoción y tiene gran utilidad e importancia hasta el día de hoy (Castañeda, 2019).

El marketing en empresas pequeñas en muchas ocasiones es manejado por el dueño del negocio o a veces es el vendedor principal o el único quien regularmente tiene poca formación o interés en ello y solo se angustia por logra el nivel de ventas que le permita mantener flujo de caja (Hoyos, 20I0).

Por todo lo expuesto se plantea el proceso de investigación buscando respuesta a la pregunta ¿Qué estrategias dentro del Marketing Mix están usando los negocios en tiempos de COVID-19?

Una posible respuesta a priori nos lleva a suponer que las estrategias de ubicación son predominantes en las empresas lo que nos lleva a plantear el objetivo de conocer cuáles son las principales estrategias utilizadas por las empresas durante el COVID-19 enmarcando las estrategias dentro de la clasificación de las 4P's.

\section{MÉTODO}

Para dar respuesta a la pregunta de investigación esté estudio tiene un enfoque mixto, por la parte cualitativa se llevan a cabo entrevistas con empresarios y por el lado cuantitativo se aplica un cuestionario con una escala Likert de 5 puntos de acuerdo con Hernández, Fernández y Baptista (2014) la investigación es un estudio con diseño y alcance descriptivo ya que busca especificar las estrategias empleadas por las empresas durante el COVID-19 por lo que es del tipo no experimental sin manipular a los sujetos bajo estudio $y$ es de cohorte Transeccional. 
Para las entrevistas los sujetos bajo estudio participaron de forma voluntaria 26 encargados de negocios en cuatro zonas, dos urbanas en Tabasco y dos en rurales uno en Tabasco y otro en Chiapas por convenir así al estudio y respetando los límites de movilidad y la sana distancia marcada por las autoridades, en la Tabla I se muestra un resumen de los tipos de negocios que se entrevistaron.

\section{Tabla I}

Tipo de negocios que participaron en entrevistas

\begin{tabular}{lc}
\hline \multicolumn{1}{c}{ Tipo de negocio } & Entrevistados \\
\hline Abarrotes & 12 \\
Comida & 3 \\
Tortillería & 3 \\
Ferretería & 2 \\
Estética & 1 \\
Farmacia & 1 \\
Servicio sastrería & 1 \\
Servicios de internet & 1 \\
Plástico & 1 \\
Papelería & 1 \\
\hline Suma & 26
\end{tabular}

Para el cuestionario se envió a grupos de negocios en Facebook y WhatsApp, donde 37 empresarios contestaron de manera voluntaria y que se pueden observar en la Tabla 2.

\section{Tabla 2}

Tipo de negocios que participaron contestaron el cuestionario

\begin{tabular}{lc}
\hline \multicolumn{1}{c}{ Tipo de negocio } & Cuestionario \\
\hline Abarrotes & $\mathrm{I}$ \\
Alimentos y bebidas & 13 \\
Belleza & 2 \\
Construcción & $\mathrm{I}$ \\
Eventos & 3 \\
Farmacia & $\mathrm{I}$ \\
Limpieza y desinfección & 2 \\
Preparación Académica & $\mathrm{I}$ \\
Productos de belleza & 1 \\
Salud & $\mathrm{I}$ \\
Seguros & 1 \\
Servicios & 9 \\
Ventas & 2 \\
\hline Suma & 37
\end{tabular}

\section{DISEÑO DE INSTRUMENTOS}

Para la entrevista se consideraron algunas cuestiones sociodemográficas y se tomaron en cuenta las 4p's del Marketing Mix, se construyó una entrevista semiestructurada que se sometió a juicio de expertos y se utilizó recogiendo también datos a través de la observación, donde cada P corresponde a una categoría de análisis.

Para el cuestionario además de las consideraciones de elementos sociodemográficos como género y nivel de estudio se construyeron 5 ítems de acuerdo con cada una de la 4P del Marketing Mix considerando las estrategias más comunes que obtuvo un Alfa de Cronbach de .855 que se considera adecuado de acuerdo con Kerlinger y Lee (2002), el instrumento se diseñó en una escala de Likert de 5 puntos que va desde totalmente de acuerdo hasta totalmente en desacuerdo.

Los datos se capturaron para posteriormente hacer el análisis en el software SPSS versión 23 versión Windows.

Aquellas cosas que limitaron la investigación fueron la disposición de tiempo por parte de los empresarios para las entrevistas, desconocimiento de la mercadotecnia y de las estrategias del Marketing Mix, interrupción de clientes durante las entrevistas.

\section{RESULTADOS}

Un primer análisis para entender como afecto la pandemia, en las ventas de las diferentes empresas, de las entrevistas se extrajo la información contenida en la Tabla 3. 


\section{Tabla 3}

Opinión de los empresarios en las ventas durante la contingencia

\section{Zona Urbana}

PJ=Fue pérdida total ya que el negocio estuvo cerrado tres meses

$\mathrm{OX}=$ ocasiono perdidas, afectando al negocio como a nosotros como líderes que cobramos comisiones por ventas

$\mathrm{KG}=$ afecto las ventas porque no genero mucho ingreso

GBonA $=$ De tener una venta diaria de $\$ 1000$, de eso se tenía un rango de ganancias del $50 \%$, es decir $\$ 500$ hoy en día solo vendo $\$ 300$ diarios GArr=Nos afectó mucho, debido a que bajaron las ventas

\begin{tabular}{ll}
\hline GFarS = debido a los horarios restringidos, las & Mab5 = No nos afectó tanto \\
ventas disminuyeron & Mab5 $=$ Como tal no nos afectó fue adaptarnos \\
ZSS= Solo disminuyeron un poco & MAb2 = se siguen vendiendo así normal \\
SS="Las ventas solo disminuyeron un poco & MFerr = seguimos vendiendo de manera normal \\
ZBell= No afecto las ventas porque & MAb4= nuestras ventas están estables
\end{tabular}
atendimos por citas

GSS= No nos afectó mucho, de hecho benefició a la empresa, ya que aumentaron los ingresos al tener mayores ventas $\mathrm{ZFib}=$ Las ventas aumentaron
MAb3= nuestras ventas de lo que tiene que ver limpieza y todo eso aumentaron

$M A b=A l$ principio nos agarró desprevenidos, hubo un momento en que no nos dimos abasto, porque las personas querían llevarse todo y también donde nos surtimos parecía que ya no nos iban a surtir.

$\mathrm{Mt}=$ viendo la necesidad las personas lo compraban
Podemos observar que los efectos fueron diversos dependiendo el giro de la empresa afectando muy poco en las zonas rurales.

\section{Tabla 4}

Estrategias identificadas en las empresas de la categoría del Marketing Mix Producto

Elemento del $\quad$ Estrategias
Marketing Mix

Producto
Tener variedad de productos para que el cliente tenga más opciones de compra.

Extender y mejorar la distribución para la entrega de sus productos.

Incluir productos de temporada como cubre-bocas, satirizantes.
Para determinar los resultados de las entrevistas se sistematizo la información en cada categoría formadas por las 4ps. En la Tabla 4 podemos observar la categoría producto y las estrategias identificadas.

Se observó que se amplió el portafolio de productos en los diferentes establecimientos debido a la demanda a causa del SAR-COVID-19 y que los establecimientos más surtidos tuvieron mayor volumen de ventas. En la Tabla 5 podemos observar el análisis de la categoría precio de los elementos del Marketing Mix. 
Tabla 5

Estrategias identificadas en las empresas de la categoría del Marketing Mix Precio

\begin{tabular}{ll}
\hline $\begin{array}{c}\text { Elemento del } \\
\text { Marketing Mix }\end{array}$ & \multicolumn{1}{c}{ Estrategias usadas } \\
\hline Precio & Reducir precios en algunos \\
& $\begin{array}{l}\text { productos por un tiempo } \\
\text { limitado. }\end{array}$ \\
& $\begin{array}{l}\text { Precios accesibles } \\
\text { Repliegue de promociones ya } \\
\text { establecidas previas a la } \\
\text { pandemia. }\end{array}$ \\
\hline
\end{tabular}

Para el caso de servicios de internet que venían manejando promociones como instalación gratis del servicio fueron retiradas debido a las necesidades de telecomunicación y uso del internet ya que se clasificaron como prioritarias. Para el caso de Abarroteras pequeñas reaccionando de acuerdo con la ley de oferta y demanda aquellas ventas bajas tuvieron que reducir precios para estar con precios por debajo de la competencia. En la Tabla 6 podemos observar el análisis de la categoría Plaza del Marketing Mix.

\section{Tabla 6}

Estrategias identificadas en las empresas de la categoría del Marketing Mix Plaza

\begin{tabular}{|c|c|}
\hline $\begin{array}{l}\text { Elemento del } \\
\text { Marketing Mix }\end{array}$ & Estrategias usadas \\
\hline Plaza & $\begin{array}{l}\text { Incluir, la entrega a domicilio } \\
\text { después de cierta cantidad de } \\
\text { compra como un nuevo servicio } \\
\text { al cliente, que le brinden mayor } \\
\text { comodidad y/o satisfacción } \\
\text { Ventas en línea } \\
\text { Ventas por medio de las redes } \\
\text { sociales } \\
\text { Ventas vía telefónica y } \\
\text { mensajería (sms y WhatsApp) } \\
\text { Ampliación de las zonas de } \\
\text { entrega. } \\
\text { Subcontratación de servicio de } \\
\text { reparto } \\
\text { Adquisición de equipo de } \\
\text { reparto como motocicletas o } \\
\text { inclusive bicicletas. }\end{array}$ \\
\hline
\end{tabular}

Por motivo de la sana distancia tuvieron que mejorar la logística de entrega de domicilio, recurriendo muchas veces al apoyo de servicios de mensajería subcontratados, algunos chicos en bicicleta se encargaban de apoyar con las entregas para lograr cumplir con el alto grado de pedidos a domicilio, estableciendo montos mínimos de venta o costos adicionales, surgiendo negocios nuevos que brindaban el servicio para satisfacer los altos grados de demanda solicitados. En la Tabla 7 podemos observar el análisis de la categoría Promoción del Marketing Mix.

\section{Tabla 7}

Estrategias identificadas en las empresas de la categoría del Marketing Mix Promoción

\begin{tabular}{ll}
\hline Elemento del & Estrategias usadas \\
Marketing Mix &
\end{tabular}

Promoción Establecer carteles, volantes, presentaciones, folletos 0 calendarios publicitarios. Organizar una estación de degustación.

Realizar sorteos de lotería o concursos entre clientes.

Crea un programa de fidelización de clientes. Creación de contenido digital para promocionar los productos.

Organizar actividades o eventos que distingan el apoyo hacia el Ejido.

Crear una página promocional.

Uso de las redes sociales

Promociones de temporada Hacer la promoción tipo $2 \times \mathrm{I} u$ obtenga recompensas adicionales al comprar el producto.

Dar regalos por comprar ciertos productos.

Servicios de intercambio de productos u otros productos requeridos por la empresa, como publicidad.

Ofertas especiales para clientes valorados 
Se observó la necesidad de verter sus catálogos en una plataforma digital para poder enviarlos a sus futuros clientes, los empresarios se vieron en la necesidad de unirse a las redes sociales como como Facebook y mercado libre que vio incrementada su actividad para colocar ventas; el uso de carteles y volantes de los negocios locales para hacer pedidos vía telefónica o por medio de mensajería instantánea como WhatsApp, siendo este último el más utilizado en las zonas; por otra parte la gran mayoría de las promociones fueron establecidas en productos que no eran de primera necesidad para aumentar la rotación de inventarios.

Respecto a los resultados arrojados por el instrumento de medición de las 38 empresas, en la Figura I podemos observar la antigüedad de los negocios participantes.

\section{Figura I}

Gráfico de pastel de acuerdo con la antigüedad del negocio

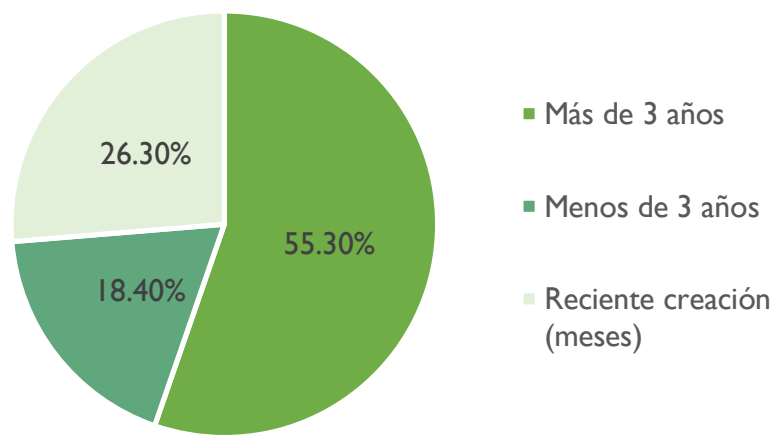

El 55\% de las empresas tiene más de 3 años de antigüedad y un $26 \%$ tiene menos de tres meses de creación, donde el $45 \%$ son de género femenino, sobre los estudios del líder un $73 \%$ tiene estudios superiores, un $40 \%$ es soltero, un $37 \%$ casado, un $10 \%$ son divorciados y el 13 restante en unión libre.
Se realizó una progresión aritmética de los ítems de cada categoría Marketing Mix para después observar los resultados descriptivos en la tabla 8 .

\section{Tabla 8}

Resultados descriptivos por cada categoría del Marketing Mix

\begin{tabular}{|c|c|c|c|c|c|}
\hline Categoría & $\mathbf{N}$ & Mínimo & Máximo & Media & D.E. \\
\hline $\begin{array}{l}\text { Total } \\
\text { Producto }\end{array}$ & 38 & 5 & 25 & 15.21 & 6.005 \\
\hline Total Precio & 38 & 5 & 22 & 12.39 & 4.600 \\
\hline Total Plaza & 38 & 5 & 25 & 18.18 & 4.909 \\
\hline $\begin{array}{l}\text { Total } \\
\text { Promoción }\end{array}$ & 38 & 5 & 23 & 13.16 & 4.577 \\
\hline $\begin{array}{l}\mathrm{N} \text { válido } \\
\text { (por lista) }\end{array}$ & 38 & & & & \\
\hline
\end{tabular}

Se puede observar que las estrategias con una media mayor son plaza con 18.18 y producto 15.21

Al buscar diferencias respecto a las medias encontradas y los datos sociodemográficos recabados por el instrumento, el género no arrojo diferencias significativas ni el grado de estudios del líder, un ANOVA de las medias poblacionales de las estrategias del Marketing Mix por categoría respecto al estado civil se pueden observar en la Tabla 9.

Se puede observar diferencias significativas en la categoría Producto y en Precio en donde la prueba post hoc Bonferroni muestra diferencias entre aquellos en unión libre respecto a los Casados y solteros en cuanto al producto donde aquellos en unión libre utilizaron menos esta estrategia, también se encontró diferencias al respecto de los de unión libre y los Solteros respecto al precio donde se puede observar que los solteros utilizaron más esta estrategia.

En la Tabla 10 podemos observar los resultados de un ANOVA entre las categorías del Marketing Mix y la antigüedad del negocio. 
Tabla 9

Comparación de medias poblaciones de las estrategias del Marketing Mix por categoría y el estado civil.

\begin{tabular}{|c|c|c|c|c|c|c|}
\hline \multicolumn{2}{|c|}{ Categoría del Marketing Mix } & \multirow{2}{*}{$\frac{N}{14}$} & \multirow{2}{*}{$\frac{\text { Media }}{16.29}$} & \multirow{2}{*}{$\frac{\text { D.E. }}{5.398}$} & \multirow{2}{*}{$\frac{\mathbf{F}}{4.542}$} & \multirow{2}{*}{$\frac{\text { Sig. }}{0.009}$} \\
\hline Total Producto & Casado & & & & & \\
\hline & Soltero & 15 & 16.93 & 5.391 & & \\
\hline & Divorciado & 4 & 15.00 & 4.830 & & \\
\hline & Unión Libre & 5 & 7.20 & 4.919 & & \\
\hline & Total & 38 & 15.21 & 6.005 & & \\
\hline \multirow[t]{5}{*}{ Total Precio } & Casado & 14 & 12.07 & 4.497 & 3.952 & 0.016 \\
\hline & Soltero & 15 & 14.27 & 4.079 & & \\
\hline & Divorciado & 4 & 13.25 & 1.258 & & \\
\hline & Unión Libre & 5 & 7.00 & 4.472 & & \\
\hline & Total & 38 & 12.39 & 4.600 & & \\
\hline \multirow[t]{5}{*}{ Total Plaza } & Casado & 14 & 17.64 & 5.943 & 0.357 & 0.785 \\
\hline & Soltero & 15 & 17.87 & 4.596 & & \\
\hline & Divorciado & 4 & 18.75 & 5.188 & & \\
\hline & Unión Libre & 5 & 20.20 & 2.683 & & \\
\hline & Total & 38 & 18.18 & 4.909 & & \\
\hline \multirow[t]{5}{*}{ Total Promoción } & Casado & 14 & 12.57 & 3.837 & 0.700 & 0.559 \\
\hline & Soltero & 15 & 13.13 & 4.422 & & \\
\hline & Divorciado & 4 & 12.00 & 7.746 & & \\
\hline & Unión Libre & 5 & 15.80 & 4.604 & & \\
\hline & Total & 38 & 13.16 & 4.577 & & \\
\hline
\end{tabular}

\section{Tabla 10}

Comparación de medias poblaciones de las estrategias del Marketing Mix por categoría y antigüedad del negocio

\begin{tabular}{|c|c|c|c|c|c|c|}
\hline \multicolumn{2}{|c|}{ Categoría del Marketing Mix } & $\mathbf{N}$ & Media & D.E. & $\mathbf{F}$ & Sig. \\
\hline \multirow[t]{4}{*}{ Total Producto } & Reciente creación & 10 & 17.00 & 5.270 & 1.128 & 0.335 \\
\hline & Menos de 3 años & 7 & 16.57 & 6.294 & & \\
\hline & Más de 3 años & 21 & 13.90 & 6.188 & & \\
\hline & Total & 38 & $|5.2|$ & 6.005 & & \\
\hline \multirow[t]{4}{*}{ Total Precio } & Reciente creación & 10 & 14.10 & 2.644 & $\mathrm{I} .464$ & 0.245 \\
\hline & Menos de 3 años & 7 & 13.29 & 4.271 & & \\
\hline & Más de 3 años & 21 & 11.29 & 5.245 & & \\
\hline & Total & 38 & 12.39 & 4.600 & & \\
\hline \multirow[t]{4}{*}{ Total Plaza } & Reciente creación & 10 & 19.80 & 4.237 & 1.937 & 0.159 \\
\hline & Menos de 3 años & 7 & 20.00 & 3.367 & & \\
\hline & Más de 3 años & 21 & 16.81 & 5.354 & & \\
\hline & Total & 38 & 18.18 & 4.909 & & \\
\hline \multirow[t]{4}{*}{ Total Promoción } & Reciente creación & 10 & 16.60 & 3.893 & 5.217 & 0.010 \\
\hline & Menos de 3 años & 7 & 10.57 & 3.823 & & \\
\hline & Más de 3 años & 21 & 12.38 & 4.318 & & \\
\hline & Total & 38 & 13.16 & 4.577 & & \\
\hline
\end{tabular}


Se puede observar en la tabla diferencias en las estrategias de promoción donde las empresas de reciente creación las aplican más, y que es comprobado en la prueba post hoc Bonferroni.

\section{CONCLUSIONES}

Dentro de las conclusiones generales basados en las observaciones in situ los establecimientos para no perder ventas la gran mayoría se vio en la necesidad de acatar las recomendaciones de la secretaría de salud sobre los cuidados preventivos con la finalidad de evitar contagios y aumentando el distanciamiento, surgió un mercado negro que atendió clandestinamente algunos productos que fueron retirados de circulación aumentando sus precios de forma indebida, otros tantos negocios se vieron en la necesidad de cerrar sus puertas debido a la baja o nula afluencia de clientes de acuerdo con el giro como en el caso del área de venta de alimentos donde algunos cerraron de manera definitiva, de igual manera muchos negocios surgieron durante la pandemia con servicios y productos que cubrieron las necesidades de protección y cuidado personal que como resulta lógico y se muestra en el cuestionario empresas de reciente creación aplican mayores promociones tratando de insertarse en el mercado.

Dentro del giro de servicios se observaron diferentes estrategias de atención a los usuarios que antes eran atendidos directamente y que se vieron en la necesidad de brindar los mismos servicios a través de video llamadas, como el caso de consultas médicas, o asesorías, servicios de animación infantil, cursos de verano y diferentes actividades deportivas (karate, ballet, etc.) sin mucho éxito varias veces debido a que muchos servicios dependían del ambiente generado por sus instalaciones y la convivencia, en otros casos con mucho éxito como servicios de nutrición al formar clubs que compartían dieta y clubs de ejercicio online al compartir rutinas.

Las conclusiones surgidas de nuestras entrevistas muestran un impacto diverso dependiendo el giro de la empresa afectando muy poco en las zonas rurales, la ampliación de portafolio de productos que atribuimos a la demanda causada por la pandemia y que los establecimientos más surtidos tuvieron mayor volumen de ventas.

Por motivo de la sana distancia tuvieron que mejorar la logística de entrega de domicilio, recurriendo muchas veces al apoyo de servicios de mensajería subcontratados, algunos chicos en bicicleta se encargaban de apoyar con las entregas para lograr cumplir con el alto grado de pedidos a domicilio, estableciendo montos mínimos de venta o costos adicionales, surgiendo negocios nuevos que brindaban el servicio para satisfacer los altos grados de demanda solicitados y dentro de nuestro cuestionario fueron las estrategias más utilizadas de acuerdo a la media 18.18.

Para el caso de servicios de internet que venían manejando promociones como instalación gratis del servicio fueron retiradas debido a las necesidades de telecomunicación y uso del internet ya que se clasificaron como prioritarias. Para el caso de Abarroteras pequeñas reaccionando de acuerdo con la ley de oferta y demanda aquellas ventas bajas tuvieron que reducir precios para estar con precios por debajo de la competencia.

Se observó la necesidad de verter sus catálogos en una plataforma digital para poder enviarlos a sus futuros clientes, los empresarios se vieron en la necesidad de unirse a las redes sociales como como 
Facebook y mercado libre que vio incrementada su actividad para colocar ventas; el uso de carteles y volantes de los negocios locales para hacer pedidos vía telefónica o por medio de mensajería instantánea como WhatsApp, siendo este último el más utilizado en las zonas; por otra parte la gran mayoría de las promociones fueron establecidas en productos que no eran de primera necesidad para aumentar la rotación de inventarios.

Un resultado lógico pero inesperado es sobre la influencia del estado civil en la decisión estratégica que en el cuestionario muestra que los solteros tienen una estrategia más agresiva sobre los precios al respecto de los que viven en unión libre y los casados, que puede ser producto de alguna necesidad psicológica y que nos deja una futura línea de investigación.

\section{REFERENCIAS}

Ansoff, H. I., y Leontiades, J. C. (1976). Strategic Portfolio Management. Journal of General Management, 4(I), I3-29. https://doi.org/I0.1 I77/030630707600400102

Ascencio, A., Campo, J., Ramírez, A., y Zapata, L. (2016). Importancia de la planeación estratégica en las áreas de gestión humana de las organizaciones. Revista Fundación Universitaria Luis Amigó, 3(I), II6.

https://doi.org/I0.2150I/238234I0.1899

Burbano-Pérez, Á. (2017). Importancia de la dirección estratégica para el desarrollo empresarial. Dominio de las Ciencias, 3(3), 19-28. https://doi.org//0.23857/dom.cien.pocaip.2017.3. monol.ago.19-28

Castañeda, J. (2019). Evolución de las 4p's o Marketing Mix. Universidad de Valladolid.

Cruz, P. (20I5). Marketing Mix. Universidad de Jaén.

García-Muñoz, C., Pérez, B., y Navarrete, M. (2020). Las Empresas ante el COVID-19. Revista GISST, 27II-2934(I), 85-10I. https://www.editorialeidec.com/revista/index.php /GISST/article/view/83/64

Gomar, R. (2020). Efectos del COVID-I 9 en la Empresa.
Hernández, R., Fernandez, C., y Baptista, P. (20/4). Metodología de la investigación. McGrawn Hill.

Hoyos, R. (2010). El papel del marketing en las Empresas: misión, objetivos y funciones. Papeles de Administración, I (6), I-18. https://repositorio.unal.edu.co/bitstream/handle/ unal/8820/ricardohoyosballesteros_2010.pdf

Instituto Nacional Electoral (abril de 2020) ACUERDO del Consejo General del Instituto Nacional Electoral por el que se determina como medida extraordinaria la suspensión de plazos inherentes a las actividades de la función electoral, con motivo de la contingencia sanitaria derivada de la pandemia del coronavirus, COVID-19. Diario Oficial de la Federación.

https://www.dof.gob.mx/nota_detalle.php?.codigo $=5590949 \&$ fecha $=0$ I $/ 04 / 2020$

Kerlinger, F., \& Lee, H. (2002). Investigación del comportamiento. Métodos de investigación en ciencias sociales. McGrawn Hill.

Marín Portillo, K., y Atencio Cárdenas, E. (2008). Proceso Académico-Administrativo de la Revista Científica y Ética del Investigador: estudio de un caso. Omnia, I4(3), I60-I83. https://www.redalyc.org/articulo.oa?id=737| | | 2 | $\underline{009}$

Matías-Guiu, J., Gomez-Pinedo, U., Montero-Escribano, P., Gomez-Iglesias, P., Porta-Etessam, J., y MatiasGuiu, J. A. (2020). ¿Es esperable que haya cuadros neurológicos por la pandemia por SARSCoV-2?. Neurología, 35 (3), 170-175. https://doi.org//0.1016/j.nrl.2020.03.001

Peñafiel-Loor, J., Pibaque-Pionce, M., y Pin-Sancan, J. (2019). La importancia de la planificación estratégica para las pequeñas y medianas empresas (PYMES). Revista Científica Ciencias Económicas y Empresariales, 4(I), I07-133. https://fipcaec.com/index.php/fipcaec/article/view II05/I4I

Pérez-Vallejo, L., Vilariño-Corella, C., y Ronda-Pupo, G. (2017). El cambio organizacional como herramienta para coadyuvar con la implementación de la estrategia. Ingeniería Industrial, 38(3), 323-332. http://www.rii.cujae.edu.cu

Ruiz, I., Aguilar, M., García, M., González, A., Vega, D., y Velandia, A. (20II). Aproximación teórica a la planeación estratégica de recursos humanos en el sector público. Administración \& Desarrollo, 39(54), 47-66.

Saldarriaga, P., y Contreras, P. (20I8). Influencia del Marketing Mix (4Ps) en el desempeño organizacional en las Empresas de seguros en el 
Perú que participen en el ramo accidentes personales y enfermedades en 2018 [Universidad San Ignacio de Loyola].

http://repositorio.usil.edu.pe/bitstream/USIL/839 9/3/2018 Saldarriaga-Coveñas.pdf

Sukier, H., Hernández-Fernández, L., Portillo-Medina, R., Valle-Ospino, A., García, M., y GarcíaGuiliany, J. (20I8). Marketing estratégico: una mirada desde el contexto de la empresa familiar. Revista ESPACIOS, 39(44).

http://www.revistaespacios.com/a I8v39n44/a I8v 39n44p09.pdf

Zabaleta, S., \& Gaona, J. (2020). ¿Cómo están afrontando la crisis las Empresas Colombianas COVID 19? 